\title{
Jackknife empirical likelihood method for case-control studies with gene-environment independence on controls
}

\author{
Bing-Yi Jing, Zhouping Li*, Jing Qin And Wang Zhou
}

In this paper, we propose a jackknife empirical likelihood method to do inference for the interested parameters of the multiplicative-intercept risk models by taking into account the gene-environment independence on controls in case-control studies. It is shown that the proposed statistic is asymptotically chi-squared distributed. Simulation studies investigate the small-sample properties. A real example is also given.

AMS 2000 SUbJeCt Classifications: Primary 62G05, 62G20; secondary 62P10.

Keywords AND Phrases: Case-control study, Geneenvironment independence, Jackknife empirical likelihood, Logistic regression, Multiplicative-intercept risk model, $U$ statistics.

\section{INTRODUCTION}

The case-control study, where sampling is conditioned on the presence (cases) and absence (controls) of a disease, is an efficient way of studying the effect of risk factors to rare diseases. As the regression coefficients have a desired interpretation in terms of log-odds ratios which are estimable based on case-control samples, logistic regression models have been widely applied to analyze binary data arising from casecontrol studies in epidemiology (see, e.g., Breslow, 1996).

To be precise, suppose that $D$ is a binary response variable of presence $(D=1)$, or absence $(D=0)$ of a disease, and $X$ is the associated explanatory variables. We consider the general logistic regression model, also known as the multiplicative-intercept risk model:

$$
P(D=1 \mid X=x)=\frac{\exp \left\{\alpha^{*}+m(x, \beta)\right\}}{1+\exp \left\{\alpha^{*}+m(x, \beta)\right\}},
$$

where $m(x, \beta)$ is a given function, $\alpha^{*}$ is a scale parameter, $\beta=\left(\beta_{1}, \ldots, \beta_{p}\right)^{T}$ is a $p$-vector parameter, and the marginal distribution of $X$ is unspecified. As in Prentice and Pyke (1979), the case-control samples are two independent groups of samples based on model (1), i.e., $X_{1}, \ldots, X_{n_{0}}$ are a random sample (controls) from $P(x \mid D=0)$ and $X_{n_{0}+1}, \ldots, X_{n}$ are another random sample (cases) from $P(x \mid D=1)$.

\footnotetext{
*Corresponding author.
}

Let $f(x \mid D=0)$ and $f(x \mid D=1)$ be the conditional density functions of the controls and cases. Under the logistic assumptions, it can be shown that

$$
f(x \mid D=1)=f(x \mid D=0) \exp \{\alpha+m(x, \beta)\},
$$

where $\alpha=\alpha^{*}+\log \{P(D=0) / P(D=1)\}$. Therefore, model (1) is equivalent to the following two-sample semiparametric density ratio model:

(2) $X_{1}, \ldots, X_{n_{0}}$ are independent with density $f(x \mid D=0)$, $X_{n_{0}+1}, \ldots, X_{n}$ are independent with density

$$
\exp \{\alpha+m(x, \beta)\} f(x \mid D=0) .
$$

Notice that model (2) is considerably flexible. For example, it is a biased sampling model with weight function $\exp \{\alpha+$ $m(x, \beta)\}$, where $\alpha$ and $\beta$ are unknown (see, e.g., Vardi, 1982; Gill et al., 1988; Qin, 1993). If $m(x, \beta)=\beta^{T} x$ with $x$ being a $p \times 1$ random vector, model (2) reduces to the standard logistic model (see, e.g., Anderson, 1979; Breslow and Day, 1980). Model (2) is also related to the Cox proportional hazards model (see, Qin, 1998).

There is extensive literature on model (2) under the casecontrol sampling plans. For example, O'Neill (1980) studied the discrimination problem in (2); Cox and Ferry (1991) and Weinberg and Wacholder (1993) investigated this model mainly for discrete data; Qin (1998) employed empirical likelihood to model (2), derived the asymptotic normality and further showed the optimality in the sense of Godambe (1960). Related work also has been done for the standard logistic regression model, references include Prentice and Pyke (1979), Anderson and Blair (1982), Wang and Carroll (1993), Qin and Zhang (1997), and Brelsow et al. (2000) among others.

Our research is motivated by epidemiological studies on gene-environment interaction problems. As the genetic susceptibility and environmental (or non-genetic) exposures play an interactive role in aetiology of many diseases, epidemiologists often seek to examine how these two risk factors affect a disease through case-control studies. Intuitively, an individual's genetic susceptibility is determined since birth, and may not relate to his/her environmental exposures. Recent studies showed that the assumption of independence between gene and environmental exposures may be reason- 
able; see Hwang et al. (1994) for example. This has attracted some researchers' attention, and they have developed new methods for analyzing case-control data in these occasions; see Piegorsch et al. (1994), Umbach and Weinberg (1997), Modan et al. (2001). However, all these methods have their own drawbacks, see Chatterjee and Carroll (2005) for more discussion. For instance, Chatterjee and Carroll (2005) considered the case-control problem when the underlying populations satisfy gene-environment independence but assume that the populations contain only finite values. Thus, seeking a novel method to this case becomes important.

As pointed out by Piegorsch et al. (1994), assumptions that genotype and exposure are independent in the population and that the disease is rare, in fact, imply that genotype and exposure are (approximately) independent in the nondiseased population. Naturally, a method that could make full use of such information will invariably increase efficiency. It is well-known that empirical likelihood approach of Owen $(1988,1990,2001)$ can easily incorporate such information in the model, in addition to its many other nice properties. In fact, the empirical likelihood to case-control studies has been investigated by Qin (1998) when there is no auxiliary information. When applying the procedure of Qin (1998) to profile out the nuisance parameters, unfortunately, this method does not produce a closed form for this problem.

To overcome the difficulties encountered by Owen's direct application of empirical likelihood, we consider the jackknife empirical likelihood (JEL) method, proposed recently by Jing, Yuan and Zhou (2009). The idea of the JEL is to construct a jackknife pseudo-values first, and then treat this jackknife pseudo-sample as a sample of i.i.d. observations and apply the standard empirical likelihood method for the mean of the jackknife pseudo-sample to obtain the empirical likelihood ratio statistic. In this paper, we study the possibility of extending the jackknife empirical likelihood method to the case-control studies taking into consideration the geneenvironment independence on controls.

We organize this paper as follows. Section 2 gives the detailed methodology and main results. A simulation study is presented in Section 3. All proofs are put in Section 4.

\section{MAIN RESULTS}

\subsection{Formulation}

Suppose that one has auxiliary information of the geneenvironment independence on controls, i.e., for $x_{i}=\left(x_{i, 1}\right.$, $x_{i, 2}$ ), we have

$$
f\left(x_{i, 1}, x_{i, 2} \mid D=0\right)=f\left(x_{i, 1} \mid D=0\right) f\left(x_{i, 2} \mid D=0\right) .
$$

Define

$$
\begin{aligned}
p_{i, 1} & =d F\left(x_{i, 1} \mid D=0\right), \quad i=1, \ldots, n_{0}, \\
p_{i, 2} & =d F\left(x_{i, 2} \mid D=0\right), \quad i=1, \ldots, n_{0}, \\
q_{k} & =d F\left(x_{k, 1}, x_{k, 2} \mid D=1\right), \quad k=n_{0}+1, \ldots, n .
\end{aligned}
$$

The log-empirical likelihood function is defined as

$$
\begin{aligned}
L(\alpha, \beta) & =\log \left(\prod_{i=1}^{n_{0}}\left(p_{i, 1} p_{i, 2}\right) \prod_{k=n_{0}+1}^{n} q_{k}\right) \\
& =\sum_{j=1}^{2} \sum_{i=1}^{n_{0}} \log p_{i, j}+\sum_{k=n_{0}+1}^{n} \log q_{k}
\end{aligned}
$$

subject to the constraints

(4)

$$
\sum_{i=1}^{n_{0}} p_{i, j}=1 \quad \text { and } \quad \sum_{k=n_{0}+1}^{n} q_{k}=1, \quad p_{i, j}, q_{k} \geq 0, j=1,2,
$$

and

$$
\begin{aligned}
& \text { (5) } \sum_{i_{1}=1}^{n_{0}} \sum_{i_{2}=1}^{n_{0}} p_{i_{1}, 1} p_{i_{2}, 2}\left[\exp \left\{\alpha+m\left(X_{i_{1}, 1}, X_{i_{2}, 2}, \beta\right)\right\}-1\right]=0 \\
& \text { (6) } \quad \sum_{k=n_{0}+1}^{n} q_{k}\left[\exp \left\{-\alpha-m\left(X_{k, 1}, X_{k, 2}, \beta\right)\right\}-1\right]=0
\end{aligned}
$$

The constraints (5) and (6) come respectively from

$$
\begin{aligned}
1 & =\int f\left(x_{i} \mid D=1\right) d x_{i}=\int \exp \left\{\alpha+m\left(x_{i}, \beta\right)\right\} d F\left(x_{i} \mid D=0\right) \\
& =\int \exp \left\{\alpha+m\left(x_{i}, \beta\right)\right\} d F\left(x_{i, 1} \mid D=0\right) d F\left(x_{i, 2} \mid D=0\right),
\end{aligned}
$$

and

$$
1=\int f\left(x_{i} \mid D=0\right) d x_{i}=\int \exp \left\{-\alpha-m\left(x_{i}, \beta\right)\right\} d F\left(x_{i} \mid D=1\right) .
$$

\subsection{Jackknife empirical likelihood}

Due to its highly nonlinear nature of the (5) and (6), direct application of Owen's empirical likelihood will encounter some serious computational difficulties. To overcome this, we consider using the jackknife empirical likelihood method, proposed by Jing, Yuan and Zhou (2009). To do this, we define $n_{1}=n-n_{0}$,

$$
\begin{aligned}
& U_{1}(\alpha, \beta)=\frac{1}{n_{0}^{2}} \sum_{i=1}^{n_{0}} \sum_{j=1}^{n_{0}} h_{1}\left(X_{i, 1}, X_{j, 2} ; \alpha, \beta\right), \\
& U_{2}(\alpha, \beta)=\frac{1}{n_{1}} \sum_{k=1}^{n_{1}} h_{2}\left(X_{n_{0}+k} ; \alpha, \beta\right)
\end{aligned}
$$

with respective kernels

$$
\begin{aligned}
h_{1}\left(X_{i, 1}, X_{j, 2} ; \alpha, \beta\right) & =\exp \left\{\alpha+m\left(X_{i, 1}, X_{j, 2}, \beta\right)\right\}-1, \\
h_{2}\left(X_{k} ; \alpha, \beta\right) & =\exp \left\{-\alpha-m\left(X_{k, 1}, X_{k, 2}, \beta\right)\right\}-1 .
\end{aligned}
$$

Note that $U_{1}(\alpha, \beta)$ is a two-sample $U$-statistic while $U_{2}(\alpha, \beta)$ is a one-sample $U$-statistic. 
However, $U_{1}(\alpha, \beta)$ can be rewritten expressed as a onesample $U$-statistic:

$$
U_{1}(\alpha, \beta)=\left(\begin{array}{c}
2 n_{0} \\
2
\end{array}\right)^{-1} \sum_{1 \leq i<j \leq 2 n_{0}} H_{1}\left(Z_{1, i}, Z_{1, j} ; \alpha, \beta\right),
$$

where

$$
\begin{aligned}
& Z_{1, i}= \begin{cases}X_{i, 1}, & i=1, \ldots, n_{0}, \\
X_{i-n_{0}, 2}, & i=n_{0}+1, \ldots, 2 n_{0},\end{cases} \\
& H_{1}\left(Z_{1, i}, Z_{1, j} ; \alpha, \beta\right) \\
& =\left\{\begin{array}{c}
\left(2 n_{0}-1\right) h_{1}\left(Z_{1, i}, Z_{1, j} ; \alpha, \beta\right) / n_{0}, \\
1 \leq i \leq n_{0}<j \leq 2 n_{0}, \\
0, \quad \text { otherwise. }
\end{array}\right.
\end{aligned}
$$

Define the jackknife pseudo-values by

$$
\begin{aligned}
& \widehat{V}_{1 i}(\alpha, \beta)=2 n_{0} U_{1}(\alpha, \beta)-\left(2 n_{0}-1\right) U_{1}^{(-i)}(\alpha, \beta), \\
& \quad i=1, \ldots, 2 n_{0}, \\
& \widehat{V}_{2 k}(\alpha, \beta)=n_{1} U_{2}(\alpha, \beta)-\left(n_{1}-1\right) U_{2}^{(-k)}(\alpha, \beta), \\
& \quad k=1, \ldots, n_{1},
\end{aligned}
$$

where $U_{1}^{(-i)}$ is calculated from $U_{1}$ using $Z_{1,1}, \ldots, Z_{1, i-1}$, $Z_{1, i+1}, \ldots, Z_{1,2 n_{0}}$ after deleting the $i$ th data value $Z_{1, i}$ while $U_{2}^{(-k)}$ is computed from $X_{n_{0}+1}, \ldots, X_{n_{0}+k-1}$, $X_{n_{0}+k+1}, \ldots, X_{n}$ after deleting the $k$ th data value $X_{n_{0}+k}$. It can be seen that

$$
\begin{aligned}
& U_{1}(\alpha, \beta)=\frac{1}{2 n_{0}} \sum_{i=1}^{2 n_{0}} \widehat{V}_{1 i}(\alpha, \beta), \\
& U_{2}(\alpha, \beta)=\frac{1}{n_{1}} \sum_{j=1}^{n_{1}} \widehat{V}_{2 j}(\alpha, \beta) .
\end{aligned}
$$

Since jackknife pseudo-values are asymptotically independent (see, Shi, 1984), we can treat $\left\{\widehat{V}_{1 i}(\alpha, \beta), i=1, \ldots\right.$, $\left.2 n_{0}\right\}$ as one sample, while $\left\{\widehat{V}_{2 i}(\alpha, \beta), i=1, \ldots, n_{1}\right\}$ (i.e., $\left.h_{2}\left(Z_{2, i} ; \alpha, \beta\right), i=1, \ldots, n_{1}\right)$ is another i.i.d. sample. Define

$p_{i}=d F\left(\widehat{V}_{1 i}(\alpha, \beta) \mid D=0\right), \quad$ and $\quad q_{j}=d F\left(\widehat{V}_{2 j}(\alpha, \beta) \mid D=1\right)$.

Then the empirical log-likelihood of those pseudo-values can be re-defined as

$$
L(\alpha, \beta)=\sum_{i=1}^{2 n_{0}} \log p_{i}+\sum_{j=1}^{n_{1}} \log q_{j}
$$

subject to the constraints

$$
\left\{\begin{array}{l}
\sum_{i=1}^{2 n_{0}} p_{i}=1, \quad \sum_{j=1}^{n_{1}} q_{j}=1, \quad p_{i}, q_{j} \geq 0 \\
\sum_{i=1}^{2 n_{0}} p_{i} \widehat{V}_{1 i}(\alpha, \beta)=0 \\
\sum_{j=1}^{n_{1}} q_{j} \widehat{V}_{2 j}(\alpha, \beta)=0 .
\end{array}\right.
$$

Using the Lagrange multiplier method, we have that $L(\alpha, \beta)$ attains its maximum at

$p_{i}=\frac{1}{2 n_{0}} \cdot \frac{1}{1+\lambda_{1} \widehat{V}_{1 i}(\alpha, \beta)}, \quad$ and $\quad q_{j}=\frac{1}{n_{1}} \cdot \frac{1}{1+\lambda_{2} \widehat{V}_{2 j}(\alpha, \beta)}$.

This, in turn, gives the $-2 \times \log$-likelihood ratio as

$$
\begin{aligned}
& l(\alpha, \beta) \\
& \quad=2 \sum_{i=1}^{2 n_{0}} \log \left\{1+\lambda_{1} \widehat{V}_{1 i}(\alpha, \beta)\right\}+2 \sum_{j=1}^{n_{1}} \log \left\{1+\lambda_{2} \widehat{V}_{2 j}(\alpha, \beta)\right\},
\end{aligned}
$$

where $\lambda_{1}$ and $\lambda_{2}$ satisfy

$$
\begin{aligned}
Q_{1 n_{0} n_{1}}\left(\alpha, \lambda_{1}, \lambda_{2}\right) & :=\frac{1}{2 n_{0}} \sum_{i=1}^{2 n_{0}} \frac{\widehat{V}_{1 i}(\alpha, \beta)}{1+\lambda_{1} \widehat{V}_{1 i}(\alpha, \beta)}=0, \\
Q_{2 n_{0} n_{1}}\left(\alpha, \lambda_{1}, \lambda_{2}\right) & :=\frac{1}{n_{1}} \sum_{j=1}^{n_{1}} \frac{\widehat{V}_{2 j}(\alpha, \beta)}{1+\lambda_{2} \widehat{V}_{2 j}(\alpha, \beta)}=0 .
\end{aligned}
$$

Since we are interested in $\beta$, we minimize $l(\alpha, \beta)$ with respect to $\alpha$ and obtain the profile likelihood ratio

$l(\beta)=2 \sum_{i=1}^{2 n_{0}} \log \left\{1+\lambda_{1} \widehat{V}_{1 i}(\hat{\alpha}, \beta)\right\}+2 \sum_{j=1}^{n_{1}} \log \left\{1+\lambda_{2} \widehat{V}_{2 j}(\hat{\alpha}, \beta)\right\}$, where $\hat{\alpha}=\arg \min _{\alpha} l(\alpha, \beta)$.

\subsection{Main results}

Before stating the main results, we list two assumptions:

Assumption 1. $\lim _{n_{0}, n_{1} \rightarrow \infty} n_{1} / 2 n_{0}=\rho<\infty$, and denote $\alpha_{0}$ and $\beta_{0}$ as the true values of $\alpha$ and $\beta$, respectively.

Assumption 2. $h_{1}\left(X_{1}, X_{2} ; \alpha, \beta_{0}\right)$ and $h_{2}\left(X ; \alpha, \beta_{0}\right)$ are bounded by some integrable function $G\left(X_{1}, X_{2}\right)$ for $\mid \alpha-$ $\alpha_{0} \mid \leq n_{0}^{-1 / 3}$.

Our main results are as follows:

Proposition 1. Assume that Assumptions 1-2 hold. Then, with probability tending to one, $l\left(\alpha, \beta_{0}\right)$ attains its minimum at some point $\hat{\alpha}$ in the interior of $\left|\alpha-\alpha_{0}\right| \leq n_{0}^{-1 / 3}$. Moreover, $\hat{\alpha}, \lambda_{1}$ and $\lambda_{2}$ in (11) satisfy (9), (10) and

$$
\begin{aligned}
& Q_{3 n_{0} n_{1}}\left(\alpha, \lambda_{1}, \lambda_{2}\right) \\
& \quad:=\frac{1}{2 n_{0}} \sum_{i=1}^{2 n_{0}} \frac{\lambda_{1} \partial \widehat{V}_{1 i}\left(\alpha, \beta_{0}\right) / \partial \alpha}{1+\lambda_{1} \widehat{V}_{1 i}\left(\alpha, \beta_{0}\right)}+\frac{1}{2 n_{0}} \sum_{j=1}^{n_{1}} \frac{\lambda_{2} \partial \widehat{V}_{2 j}\left(\alpha, \beta_{0}\right) / \partial \alpha}{1+\lambda_{2} \widehat{V}_{2 j}\left(\alpha, \beta_{0}\right)} \\
& \quad=0 .
\end{aligned}
$$

Theorem 1. Assume that Assumptions 1-2 hold. We have $l\left(\hat{\alpha}, \beta_{0}\right) \stackrel{d}{\rightarrow} \chi_{1}^{2}$, as $n \rightarrow \infty$, where $\hat{\alpha}$ is given in Proposition 1. 
From Theorem 1, a confidence interval of $\beta_{0}$ with level $\gamma$ can be obtained as

$$
I_{\gamma}(r)=\left\{\beta: l(\hat{\alpha}, \beta) \leq \chi_{1, \gamma}^{2}\right\},
$$

where $\chi_{1, \gamma}^{2}$ is the $\gamma$ th quantile of $\chi_{1}^{2}$.

\section{SIMULATIONS}

In this section, we conduct a simulation study to evaluate the finite sample performances of the proposed jackknife empirical likelihood confidence interval for the interested parameters. We consider the following experiment, the components of the controls are independent with $X_{1,1} \sim N\left(\mu_{1},(1-\right.$ $\left.\left.\rho^{2}\right) \sigma_{1}^{2}\right)$ and $X_{1,2} \sim N\left(\mu_{2},\left(1-\rho^{2}\right) \sigma_{2}^{2}\right)$, while two components of the cases are not independent and follow a bivariate normal distribution, i.e., $\left(Y_{1,1}, Y_{1,2}\right) \sim B N\left(\mu_{1}, \mu_{2}, \sigma_{1}^{2}, \sigma_{2}^{2}, \rho\right)$. That is,

$$
\begin{aligned}
f\left(x_{1}, x_{2} \mid D=0\right)= & \frac{1}{\sqrt{2 \pi} \sigma_{1} \sqrt{1-\rho^{2}}} \exp \left\{-\frac{\left(x_{1}-\mu_{1}\right)^{2}}{2\left(1-\rho^{2}\right) \sigma_{1}^{2}}\right\} \\
& \times \frac{1}{\sqrt{2 \pi} \sigma_{2} \sqrt{1-\rho^{2}}} \exp \left\{-\frac{\left(x_{2}-\mu_{2}\right)^{2}}{2\left(1-\rho^{2}\right) \sigma_{2}^{2}}\right\}, \\
f\left(x_{1}, x_{2} \mid D=1\right)= & \exp \{\alpha+m(x, \beta)\} f\left(x_{1}, x_{2} \mid D=0\right),
\end{aligned}
$$

where

$$
\begin{aligned}
\alpha= & \log \sqrt{1-\rho^{2}}+\frac{\rho \mu_{1} \mu_{2}}{\left(1-\rho^{2}\right) \sigma_{1} \sigma_{2}}, \\
m\left(x_{1}, x_{2}, \beta\right)= & \beta_{1} x_{1}+\beta_{2} x_{2}+\beta_{3} x_{1} x_{2} \\
= & -\frac{\rho \mu_{2}}{\left(1-\rho^{2}\right) \sigma_{1} \sigma_{2}} x_{1}-\frac{\rho \mu_{1}}{\left(1-\rho^{2}\right) \sigma_{1} \sigma_{2}} x_{2} \\
& +\frac{\rho}{\left(1-\rho^{2}\right) \sigma_{1} \sigma_{2}} x_{1} x_{2} .
\end{aligned}
$$

In our simulation, we consider $\rho=0.2$ and 0.5 . For each setup, we put $\mu_{1}=1, \mu_{2}=0.5, \sigma_{1}=\sigma_{2}=1$, and generate 1,000 cases and 1,000 controls with sample sizes $n_{0}=n_{1}=$ $20,40,70$, respectively. We employ the package 'emplik' in the software $R$ to compute the coverage probabilities of the proposed jackknife empirical likelihood method.

For comparisons, we also computed the coverage probabilities of the logistic regression method not using the independent information in controls while they actually have, i.e., all of the setup are the same as before, and the cases and controls are corresponding to 1 and 0 , respectively, we employ the $R$ package "glm" to do the logistic regression. Thus, the confidence region for $\beta=\left(\beta_{1}, \beta_{2}, \beta_{3}\right)$ with confidence level $\gamma$ is given by

$$
\left\{\hat{\beta}:\left(\hat{\beta}-\beta_{0}\right)^{T} \hat{\Sigma}^{-1}\left(\hat{\beta}-\beta_{0}\right) \leq \chi_{3, \gamma}^{2}\right\},
$$

where $\chi_{3, \gamma}^{2}$ is the $\gamma$ th quantile of $\chi_{3}^{2} . \hat{\beta}=\left(\hat{\beta}_{1}, \hat{\beta}_{2}, \hat{\beta}_{3}\right)$ and the variance-covariance matrix $\hat{\Sigma}$ for $\hat{\beta}$ can be obtained through the package simultaneously.

In Table 1, we report the coverage probabilities of the $90 \%$ and $95 \%$ confidence regions for $\beta_{0}$ using JEL and logistic
Table 1. Coverage probabilities of the $90 \%$ and $95 \%$ confidence intervals for $\left(\beta_{1}, \beta_{2}, \beta_{3}\right)$

\begin{tabular}{lcccc}
\hline \hline$\left.n_{0}, n_{1}, \rho\right)$ & \multicolumn{2}{c}{ JEL } & \multicolumn{2}{c}{ Logistic } \\
\cline { 2 - 5 } \cline { 4 - 5 } & $\gamma=0.90$ & $\gamma=0.95$ & $\gamma=0.90$ & $\gamma=0.95$ \\
\hline$(20,20,0.2)$ & 0.863 & 0.927 & 0.965 & 0.991 \\
$(20,20,0.5)$ & 0.865 & 0.914 & 0.957 & 0.989 \\
$(40,40,0.2)$ & 0.877 & 0.937 & 0.934 & 0.977 \\
$(40,40,0.5)$ & 0.865 & 0.927 & 0.919 & 0.967 \\
$(70,70,0.2)$ & 0.882 & 0.938 & 0.912 & 0.968 \\
$(70,70,0.5)$ & 0.901 & 0.952 & 0.889 & 0.954 \\
\hline
\end{tabular}

regression. From these simulation results, we observe:

- As sample size increases, the coverage probabilities in both cases converge to the nominal levels.

- The JEL method performs better than logistic regression method which does not use the independence among controls in most cases.

- The JEL tends to have under-coverage probabilities while the logistic regression tend to have over-coverage probabilities. However, over-coverage probabilities are usually achieved by too wide confidence regions, which are less desirable.

\section{A REAL EXAMPLE}

We apply our method to the data set of Cameron and Pauling (1978). See Table 33.1 in Andrew and Herzberg (1985). The survival times of 100 terminal cancer patients (cases) and 1,000 matched controls were recorded. To be specific, the treated group of 100 patients with terminal cancer of various kinds began ascorbate treatment, while the controls received the same treatment as the treated group except for the ascorbate. This data set comprises the survival times of the ascorbate-treated patients after the date of first hospital attendance for the cancer that became untreatable, their survival times measured from the dates of untreatability, and the corresponding mean values for the matched controls. We are interested in whether supplemental ascorbate prolongs the survival times of patients with terminal cancer, and we use JEL to estimate the parameters involved. We consider $m(x, \beta)=m\left(x_{1}, x_{2}, \beta\right)$ in (1) as

$$
m(x, \beta)=\beta_{1} x_{1}+\beta_{2} x_{2}+\beta_{3} x_{1} x_{2}+\beta_{4} x_{1}^{2} .
$$

References on density ratio model selection include Qin and Zhang (1997), Fokianos (2007), among others. The survival times of patients of stomach cancer and breast cancer (Table 33.1) were selected for illustrating purposes. Here we transform the survival times in days to years, i.e., $/ 365$, and exclude two patients who were alive on 15 May 1978.

From Table 2, we observe that the confidence intervals of $\beta_{3}$ do not include 0 . Then, the supplemental ascorbate impacts the survival times of patients with terminal human 
Table 2. $90 \%$ and $95 \%$ confidence intervals for $\left(\beta_{1}, \beta_{2}, \beta_{3}, \beta_{4}\right)$

\begin{tabular}{lll}
\hline \hline & \multicolumn{2}{c}{ I } \\
\hline \multirow{3}{*}{$\beta$} & \multicolumn{2}{c}{ Stomach } \\
\cline { 2 - 3 } & $\gamma=0.90$ & \multicolumn{2}{c}{$\gamma=0.95$} \\
\hline$\beta_{1}$ & $(-59.24426,-58.34373)$ & $(-59.28645,-57.86839)$ \\
$\beta_{2}$ & $(70.54456,71.44511)$ & $(70.50234,71.92042)$ \\
$\beta_{3}$ & $(-14.94648,-14.04593)$ & $(-14.98869,-13.57061)$ \\
$\beta_{4}$ & $(17.56186,18.46241)$ & $(17.51964,18.93772)$ \\
\hline \multicolumn{2}{c}{ II } \\
\hline \multicolumn{2}{c}{ Breast } \\
\cline { 2 - 3 } & \multicolumn{2}{c}{$\gamma=0.95$} \\
\hline$\beta_{1}$ & $(-4.0041133,-3.9136229)$ & $(-4.0065935,-3.9077849)$ \\
$\beta_{2}$ & $(13.1403123,13.2308033)$ & $(13.1378322,13.2366412)$ \\
$\beta_{3}$ & $(-1.6815360,-1.5910451)$ & $(-1.6840162,-1.5852071)$ \\
$\beta_{4}$ & $(0.6463878,0.7368786)$ & $(0.6439076,0.7427167)$ \\
\hline
\end{tabular}

cancer. So, the proposed method is helpful for practical data analysis in case control studies.

\section{PROOFS}

For simplicity, we give some notations. Put

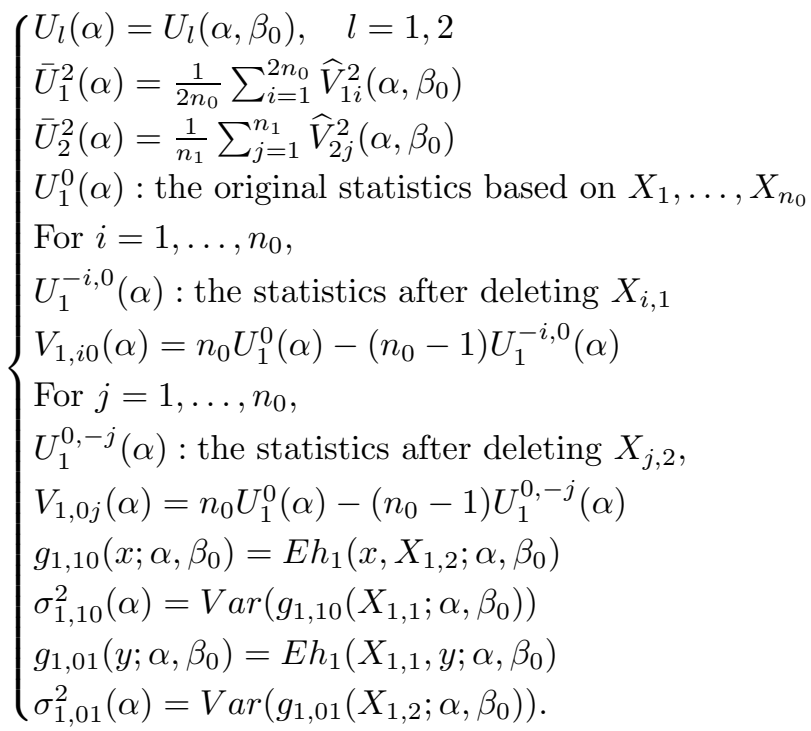

It is known that the consistent estimator of $\operatorname{Var}\left(U_{1}(\alpha)\right)$ is (see Arvesen, 1969)

$$
\begin{aligned}
\widehat{\operatorname{Var}}_{J a c k}\left(U_{1}(\alpha)\right)= & \frac{1}{n_{0}\left(n_{0}-1\right)} \sum_{i=1}^{n_{0}}\left(V_{1, i 0}(\alpha)-\bar{V}_{1, \bullet 0}(\alpha)\right)^{2} \\
& +\frac{1}{n_{0}\left(n_{0}-1\right)} \sum_{j=1}^{n_{0}}\left(V_{1,0 j}(\alpha)-\bar{V}_{1,0 \bullet}(\alpha)\right)^{2}
\end{aligned}
$$

where $\bar{V}_{1, \bullet 0}(\alpha)$ and $\bar{V}_{1,0 \bullet}(\alpha)$ are the averages of $V_{1, i 0}(\alpha)$ and $V_{1,0 i}(\alpha)$, respectively. From Schechtman and Schecht- man (2002), we have

$$
\bar{V}_{1, \bullet 0}(\alpha)=U_{1}(\alpha), \quad \bar{V}_{1,0 \bullet}(\alpha)=U_{1}(\alpha) .
$$

For $i=1, \ldots, 2 n_{0}, j=1, \ldots, n_{1}$, it can be verified that

$$
\frac{\partial \widehat{V}_{1 i}(\alpha)}{\partial \alpha}=\widehat{V}_{1 i}(\alpha)+1, \quad \frac{\partial \widehat{V}_{2 j}(\alpha)}{\partial \alpha}=-\widehat{V}_{2 j}(\alpha)-1 .
$$

First we list some lemmas.

Lemma 1. Under the conditions of Proposition 1, and if $\sigma_{1,10}^{2}(\alpha), \sigma_{1,01}^{2}(\alpha)>0$, for each $\alpha$ such that $\left|\alpha-\alpha_{0}\right| \leq n_{0}^{-1 / 3}$. Then as $n_{0}, n_{1} \rightarrow \infty$, we have

$$
\left\{\begin{array}{l}
n_{0}^{1 / 2} U_{1}\left(\alpha_{0}\right) \stackrel{d}{\rightarrow} N\left(0, S_{1}^{2}\right) \\
n_{1}^{1 / 2} U_{2}\left(\alpha_{0}\right) \stackrel{d}{\rightarrow} N\left(0, S_{2}^{2}\right) \\
\bar{U}_{1}^{2}\left(\alpha_{0}\right)=S_{1}^{2}+o_{p}(1) \\
\bar{U}_{2}^{2}\left(\alpha_{0}\right)=S_{2}^{2}+o_{p}(1),
\end{array}\right.
$$

where $S_{1}^{2}=2\left(\sigma_{1,10}^{2}\left(\alpha_{0}\right)+\sigma_{1,01}^{2}\left(\alpha_{0}\right)\right), S_{2}^{2}=E h_{2}^{2}\left(X_{n_{0}+1}\right.$; $\left.\alpha_{0}, \beta_{0}\right)$.

Proof. The first one is from Arvesen (1969), while the second one comes from the central limit theorem. Now, we prove that $\bar{U}_{1}^{2}\left(\alpha_{0}\right)=S_{1}^{2}+o_{p}(1)$. For $i=1, \ldots, n_{0}$, it can be checked that

$$
\begin{aligned}
\frac{1}{n_{0}} & \sum_{i=1}^{n_{0}} \widehat{V}_{1 i}^{2}\left(\alpha_{0}\right) \\
& =\frac{1}{n_{0}} \sum_{i=1}^{n_{0}}\left[\frac{2 n_{0}-1}{n_{0}-1} V_{1, i 0}\left(\alpha_{0}\right)-\frac{n_{0}}{n_{0}-1} U_{1}\left(\alpha_{0}\right)\right]^{2} \\
& =\left(\frac{2 n_{0}-1}{n_{0}-1}\right)^{2} \frac{1}{n_{0}} \sum_{i=1}^{n_{0}}\left[V_{1, i 0}\left(\alpha_{0}\right)-U_{1}\left(\alpha_{0}\right)\right]^{2}+U_{1}^{2}\left(\alpha_{0}\right),
\end{aligned}
$$

when $i=n_{0}+1, \ldots, 2 n_{0}$,

$$
\begin{aligned}
\frac{1}{n_{0}} & \sum_{i=n_{0}+1}^{2 n_{0}} \widehat{V}_{1\left(i-n_{0}\right)}^{2}\left(\alpha_{0}\right) \\
= & \frac{1}{n_{0}} \sum_{i=n_{0}+1}^{2 n_{0}}\left[\frac{2 n_{0}-1}{n_{0}-1} V_{1,0\left(i-n_{0}\right)}\left(\alpha_{0}\right)-\frac{n_{0}}{n_{0}-1} U_{1}\left(\alpha_{0}\right)\right]^{2} \\
= & \left(\frac{2 n_{0}-1}{n_{0}-1}\right)^{2} \frac{1}{n_{0}} \sum_{i=n_{0}+1}^{2 n_{0}}\left[V_{1,0\left(i-n_{0}\right)}\left(\alpha_{0}\right)-U_{1}\left(\alpha_{0}\right)\right]^{2} \\
& +U_{1}^{2}\left(\alpha_{0}\right) .
\end{aligned}
$$

Thus,

$$
\begin{aligned}
\bar{U}_{1}^{2}(\alpha)= & \frac{1}{2} \frac{\left(2 n_{0}-1\right)^{2}}{n_{0}-1}\left\{\frac{1}{n_{0}\left(n_{0}-1\right)} \sum_{i=1}^{n_{0}}\left[V_{1, i 0}\left(\alpha_{0}\right)-U_{1}\left(\alpha_{0}\right)\right]^{2}\right. \\
& \left.+\frac{1}{n_{0}\left(n_{0}-1\right)} \sum_{i=n_{0}+1}^{2 n_{0}}\left[V_{1,0\left(i-n_{0}\right)}\left(\alpha_{0}\right)-U_{1}\left(\alpha_{0}\right)\right]^{2}\right\}
\end{aligned}
$$




$$
\begin{aligned}
& +U_{1}^{2}\left(\alpha_{0}\right) \\
= & 2\left(n_{0}-1\right) \widehat{\operatorname{Var}}_{J a c k}\left(U_{1}\left(\alpha_{0}\right)\right)+o_{p}(1) \\
= & S_{1}^{2}+o_{p}(1),
\end{aligned}
$$

where the last equality comes from the fact that $\widehat{\operatorname{Var}}_{J a c k}\left(U_{1}\left(\alpha_{0}\right)\right)-n_{0}^{-1}\left(\sigma_{1,10}^{2}\left(\alpha_{0}\right)+\sigma_{1,01}^{2}\left(\alpha_{0}\right)\right)=o_{p}\left(n_{0}^{-1}\right)$. Finally,

$$
\begin{aligned}
\bar{U}_{2}^{2}\left(\alpha_{0}\right) & =\frac{1}{n_{1}} \sum_{j=1}^{n_{1}} \widehat{V}_{2 j}^{2}\left(\alpha_{0}\right)=\frac{1}{n_{1}} \sum_{j=1}^{n_{1}} h_{2}^{2}\left(X_{n_{0}+j} ; \alpha_{0}, \beta_{0}\right) \\
& \stackrel{p}{\rightarrow} E h_{2}^{2}\left(X_{n_{0}+1} ; \alpha_{0}, \beta_{0}\right) .
\end{aligned}
$$

Hence, we complete the proof.

Lemma 2. Under conditions of Proposition 1, if $\sigma_{1,10}^{2}(\alpha)$, $\sigma_{1,01}^{2}(\alpha)>0$, for each $\alpha \in \Theta\left(\alpha_{0}\right)$, then as $n \rightarrow \infty$, we have

$$
\begin{gathered}
P\left(\min _{1 \leq i \leq 2 n_{0}} \widehat{V}_{1 i}(\alpha)<0<\max _{1 \leq i \leq 2 n_{0}} \widehat{V}_{1 i}(\alpha)\right) \rightarrow 1, \\
P\left(\min _{1 \leq j \leq n_{1}} \widehat{V}_{2 j}(\alpha)<0<\max _{1 \leq j \leq n_{1}} \widehat{V}_{2 j}(\alpha)\right) \rightarrow 1 .
\end{gathered}
$$

Proof. Note that

$$
\begin{aligned}
\widehat{V}_{1 i}(\alpha)= & \frac{2 n_{0}-1}{n_{0}-1}\left\{V_{1, i 0}(\alpha) I\left(1 \leq i \leq n_{0}\right)\right. \\
& \left.+V_{1,0(i-n)}(\alpha) I\left(n_{0}+1 \leq i \leq 2 n_{0}\right)\right\}-\frac{n_{0}}{n_{0}-1} U_{1}(\alpha), \\
\widehat{V}_{2 j}(\alpha)= & h_{2}\left(X_{n_{0}+j} ; \alpha_{0}, \beta_{0}\right) \\
= & \exp \left\{-\alpha_{0}-m\left(X_{n_{0}+j}, \beta\right)\right\}-1 .
\end{aligned}
$$

Following similar arguments to those of Jing et al. (2009), we can reach the conclusion.

Lemma 3. Under the conditions of Proposition 1, we have

$$
\left\{\begin{array}{l}
U_{1}(\alpha)=O_{p}\left(n_{0}^{-1 / 3}\right) \\
U_{2}(\alpha)=O_{p}\left(n_{0}^{-1 / 3}\right) \\
\bar{U}_{1}^{2}(\alpha)=S_{1}^{2}+o_{p}(1) \\
\bar{U}_{2}^{2}(\alpha)=S_{2}^{2}+o_{p}(1),
\end{array}\right.
$$

where $O_{p}(\cdot)$ and $o_{p}(\cdot)$ hold uniformly for $\alpha \in\left\{\alpha:\left|\alpha-\alpha_{0}\right| \leq\right.$ $\left.n_{0}^{-1 / 3}\right\}$.

Proof. We only prove the cases of $U_{1}(\alpha)$ and $\bar{U}_{1}(\alpha)$. Applying Taylor expansion, and noting the fact (13), we have

$$
U_{1}(\alpha)=\frac{1}{2 n_{0}} \sum_{i=1}^{2 n_{0}} \widehat{V}_{1 i}\left(\alpha_{0}\right)+\frac{1}{2 n_{0}} \sum_{i=1}^{2 n_{0}}\left[\widehat{V}_{1 i}\left(\alpha^{*}\right)+1\right]\left(\alpha-\alpha_{0}\right),
$$

where $\alpha^{*}$ is between $\alpha$ and $\alpha_{0}$. As

$$
\frac{1}{2 n_{0}} \sum_{i=1}^{2 n_{0}} \widehat{V}_{1 i}\left(\alpha^{*}\right)=\frac{1}{n_{0}^{2}} \sum_{i=1}^{n_{0}} \sum_{j=1}^{n_{0}} h_{1}\left(X_{i, 1}, X_{j, 2} ; \alpha^{*}, \beta_{0}\right)
$$

298 B.-Y. Jing et al.

$$
\begin{aligned}
& \leq \frac{1}{n_{0}^{2}} \sum_{i=1}^{n_{0}} \sum_{j=1}^{n_{0}} G\left(X_{i, 1}, X_{j, 2}\right) \\
& \stackrel{p}{\rightarrow} E\left[G\left(X_{1,1}, X_{1,2}\right)\right]<\infty,
\end{aligned}
$$

this together with Lemma 1 leads to $U_{1}(\alpha)=O_{p}\left(n_{0}^{-1 / 3}\right)$. Next, let's look at $\bar{U}_{1}^{2}(\alpha)$. Notice that

$$
\begin{aligned}
\left|\bar{U}_{1}^{2}(\alpha)-\bar{U}_{1}^{2}\left(\alpha_{0}\right)\right| \\
=\left|\frac{1}{n_{0}} \sum_{i=1}^{2 n_{0}} \widehat{V}_{1 i}\left(\alpha^{*}\right)\left[\widehat{V}_{1 i}\left(\alpha^{*}\right)+1\right]\left(\alpha-\alpha_{0}\right)\right| \\
\leq\left|\frac{1}{n_{0}} \sum_{i=1}^{n_{0}} \widehat{V}_{1 i}\left(\alpha^{*}\right)\left[\widehat{V}_{1 i}\left(\alpha^{*}\right)+1\right]\left(\alpha-\alpha_{0}\right)\right| \\
\quad+\left|\frac{1}{n_{0}} \sum_{i=n_{0}+1}^{2 n_{0}} \widehat{V}_{1 i}\left(\alpha^{*}\right)\left[\widehat{V}_{1 i}\left(\alpha^{*}\right)+1\right]\left(\alpha-\alpha_{0}\right)\right| \\
:=A_{1}+A_{2} .
\end{aligned}
$$

Now,

$$
\begin{aligned}
A_{1} & =\left|\frac{1}{n_{0}} \sum_{i=1}^{n_{0}} \widehat{V}_{1 i}\left(\alpha^{*}\right)\left[\widehat{V}_{1 i}\left(\alpha^{*}\right)+1\right]\left(\alpha-\alpha_{0}\right)\right| \\
& \leq\left|\frac{1}{n_{0}} \sum_{i=1}^{n_{0}} \widehat{V}_{1 i}^{2}\left(\alpha^{*}\right)\left(\alpha-\alpha_{0}\right)\right|+\left|\frac{1}{n_{0}} \sum_{i=1}^{n_{0}} \widehat{V}_{1 i}\left(\alpha^{*}\right)\left(\alpha-\alpha_{0}\right)\right| \\
& =\left|\frac{1}{n_{0}} \sum_{i=1}^{n_{0}} \widehat{V}_{1 i}^{2}\left(\alpha^{*}\right)\right| O_{p}\left(n_{0}^{-1 / 3}\right)+O_{p}(1) O_{p}\left(n_{0}^{-1 / 3}\right),
\end{aligned}
$$

while

$$
\begin{aligned}
& \left|\frac{1}{n_{0}} \sum_{i=1}^{n_{0}} \widehat{V}_{1 i}^{2}\left(\alpha^{*}\right)\right| \\
& =\mid \frac{1}{n_{0}} \sum_{i=1}^{n_{0}}\left\{\frac{1}{n_{0}^{2}\left(n_{0}-1\right)^{2}}\right. \\
& \times\left[\sum_{i_{1}=1}^{n_{0}} \sum_{i_{2}=1}^{n_{0}} h_{1}\left(X_{i_{1}, 1}, X_{i_{2}, 2} ; \alpha^{*}, \beta_{0}\right)\right]^{2} \\
& +\frac{\left(2 n_{0}-1\right)^{2}}{n_{0}^{2}\left(n_{0}-1\right)^{2}}\left[\sum_{i_{2}=1}^{n_{0}} h_{1}\left(X_{i, 1}, X_{i_{2}, 2} ; \alpha^{*}, \beta_{0}\right)\right]^{2} \\
& -\frac{2\left(2 n_{0}-1\right)}{n_{0}^{2}\left(n_{0}-1\right)^{2}} \sum_{i_{2}=1}^{n_{0}} h_{1}\left(X_{i, 1}, X_{i_{2}, 2} ; \alpha^{*}, \beta_{0}\right) \\
& \left.\times \sum_{i_{1}=1}^{n_{0}} \sum_{i_{2}=1}^{n_{0}} h_{1}\left(X_{i_{1}, 1}, X_{i_{2}, 2} ; \alpha^{*}, \beta_{0}\right)\right\} \mid \\
& \leq 4\left[\frac{1}{n_{0}^{2}} \sum_{i_{1}=1}^{n_{0}} \sum_{i_{2}=1}^{n_{0}} h_{1}\left(X_{i_{1}, 1}, X_{i_{2}, 2} ; \alpha^{*}, \beta_{0}\right)\right]^{2} \\
& +\frac{9}{n_{0}} \sum_{i=1}^{n_{0}}\left[\frac{1}{n_{0}} \sum_{i_{2}=1}^{n_{0}} h_{1}\left(X_{i, 1}, X_{i_{2}, 2} ; \alpha^{*}, \beta_{0}\right)\right]^{2}
\end{aligned}
$$




$$
\begin{aligned}
& \leq \frac{4}{n_{0}^{2}} \sum_{i_{1}=1}^{n_{0}} \sum_{i_{2}=1}^{n_{0}} h_{1}^{2}\left(X_{i_{1}, 1}, X_{i_{2}, 2} ; \alpha^{*}, \beta_{0}\right) \\
& \quad+\frac{9}{n_{0}} \sum_{i=1}^{n_{0}} \frac{1}{n_{0}} \sum_{i_{2}=1}^{n_{0}} h_{1}^{2}\left(X_{i, 1}, X_{i_{2}, 2} ; \alpha^{*}, \beta_{0}\right) \\
& \leq C \frac{1}{n_{0}^{2}} \sum_{i_{1}=1}^{n_{0}} \sum_{i_{2}=1}^{n_{0}} G\left(X_{i_{1}, 1}, X_{i_{2}, 2}\right) \\
& \stackrel{p}{\rightarrow} C E\left(G\left(X_{i_{1}, 1}, X_{i_{2}, 2}\right)\right)<\infty .
\end{aligned}
$$

Here, just use $(a+b)^{2} \leq 2\left(a^{2}+b^{2}\right)$. Hence, $A_{1}=O_{p}\left(n_{0}^{-1 / 3}\right)$. Similarly, we can prove that $A_{2}=O_{p}\left(n_{0}^{-1 / 3}\right)$, which in turn implies $\bar{U}_{1}^{2}(\alpha)=\bar{U}_{1}^{2}\left(\alpha_{0}\right)+O_{p}\left(n_{0}^{-1 / 3}\right)$.

Lemma 4. Let $J_{1}(\alpha)=\max _{1 \leq i \neq j \leq 2 n_{0}}\left|h_{1}\left(X_{i}, X_{j} ; \alpha, \beta_{0}\right)\right|$, and $J_{2}(\alpha)=\max _{1 \leq i \leq n_{1}}\left|h_{2}\left(X_{n_{0}+i} ; \alpha, \beta_{0}\right)\right|$. Then, under the conditions of Proposition 1, we have $J_{l}=o_{p}\left(n_{0}^{1 / 3}\right)$, uniformly in $\alpha \in\left\{\alpha:\left|\alpha-\alpha_{0}\right| \leq n_{0}^{-1 / 3}\right\}$.

Proof. We only prove that $J_{1}(\alpha)=o_{p}\left(n_{0}^{1 / 3}\right)$. By the chain argument, it suffices to prove that $3^{-n_{0} / 3} \times$ $\max _{1 \leq j<3^{n_{0}}}\left|h_{1}\left(X_{j, 1}, X_{3^{n_{0}, 2}} ; \alpha, \beta_{0}\right)\right| \stackrel{p}{\rightarrow} 0$. For each $\epsilon>0$, we have

$$
\begin{aligned}
\sum_{n_{0}=1}^{\infty} & P\left(\max _{\alpha} \max _{1 \leq j<3^{n_{0}}}\left|h_{1}\left(X_{j, 1}, X_{3^{n_{0}}, 2} ; \alpha, \beta_{0}\right)\right| \geq \epsilon 3^{n_{0} / 3}\right) \\
\leq & \sum_{n_{0}=1}^{\infty} 3^{n_{0}} P\left(\max _{\alpha}\left|h_{1}\left(X_{j, 1}, X_{3^{n_{0}, 2}} ; \alpha, \beta_{0}\right)\right| \geq \epsilon 3^{n_{0} / 3}\right) \\
= & \sum_{n_{0}=1}^{\infty} \sum_{m=n_{0}}^{\infty} 3^{n_{0}} P\left(3^{(m+1) / 3}>\epsilon^{-1}\right. \\
& \left.\times \max _{\alpha}\left|h_{1}\left(X_{j, 1}, X_{3^{n_{0}}, 2} ; \alpha, \beta_{0}\right)\right| \geq 3^{m / 3}\right) \\
= & \sum_{m=1}^{\infty} \sum_{n_{0}=1}^{m} 3^{n_{0}} P\left(3^{(m+1) / 3}>\epsilon^{-1}\right. \\
& \left.\times \max _{\alpha}\left|h_{1}\left(X_{j, 1}, X_{3^{n_{0}, 2}} ; \alpha, \beta_{0}\right)\right| \geq 3^{m / 3}\right) \\
\leq & \sum_{m=1}^{\infty} 3^{m+1} P\left(3^{(m+1) / 3}>\epsilon^{-1}\right. \\
& \left.\times \max _{\alpha}\left|h_{1}\left(X_{j, 1}, X_{3^{n_{0}, 2}} ; \alpha, \beta_{0}\right)\right| \geq 3^{m / 3}\right) \\
\leq & 3 \epsilon^{-3} E \max _{\alpha}\left|h_{1}\left(X_{j, 1}, X_{3^{n_{0}}, 2} ; \alpha, \beta_{0}\right)\right|^{3} \\
\leq & 3 \epsilon^{-3} E G\left(X_{1,1}, X_{1,2}\right)<\infty
\end{aligned}
$$

Now, by the Borel-Cantelli lemma, with probability one, we have

$$
3^{-n_{0} / 3} \max _{1 \leq j<3^{n_{0}}}\left|h_{1}\left(X_{j, 1}, X_{3^{n_{0}}, 2} ; \alpha, \beta_{0}\right)\right| \rightarrow 0
$$

uniformly holds in $\alpha$.
Combining Lemmas 3 and 4, we have

$$
\left\{\begin{array}{l}
\max _{1 \leq i \leq 2 n_{0}}\left|\widehat{V}_{1 i}\left(\alpha, \beta_{0}\right)\right|=o_{p}\left(n_{0}^{1 / 3}\right) \\
\max _{1 \leq j \leq n_{1}}\left|\widehat{V}_{2 j}\left(\alpha, \beta_{0}\right)\right|=o_{p}\left(n_{0}^{1 / 3}\right) \\
\frac{1}{2 n_{0}} \sum_{i=1}^{2 n_{0}}\left|\widehat{V}_{1 i}\left(\alpha, \beta_{0}\right)\right|^{3}=o_{p}\left(n_{0}^{1 / 3}\right) \\
\frac{1}{n_{1}} \sum_{j=1}^{n_{1}}\left|\widehat{V}_{2 j}\left(\alpha, \beta_{0}\right)\right|^{3}=o_{p}\left(n_{0}^{1 / 3}\right),
\end{array}\right.
$$

all hold uniformly in $\alpha$.

Lemma 5. For $\lambda_{1}=\lambda_{1}(\alpha)$ and $\lambda_{2}=\lambda_{2}(\alpha)$ satisfying (9) and (10), under conditions of Proposition 1, we have

$$
\lambda_{l}=O_{p}\left(n_{0}^{-1 / 3}\right), \quad l=1,2,
$$

uniformly for $\left\{\alpha:\left|\alpha-\alpha_{0}\right| \leq n_{0}^{-1 / 3}\right\}$.

Proof. From (9), we have

$$
\begin{aligned}
0 & =\left|\frac{1}{2 n_{0}} \sum_{i=1}^{2 n_{0}} \frac{\widehat{V}_{1 i}\left(\alpha, \beta_{0}\right)}{1+\lambda_{1} \widehat{V}_{1 i}\left(\alpha, \beta_{0}\right)}\right| \\
& \geq \frac{1}{2 n_{0}}\left|\sum_{i=1}^{2 n_{0}} \frac{\lambda_{1} \widehat{V}_{1 i}^{2}\left(\alpha, \beta_{0}\right)}{1+\lambda_{1} \widehat{V}_{1 i}\left(\alpha, \beta_{0}\right)}\right|-\left|\frac{1}{2 n_{0}} \sum_{i=1}^{2 n_{0}} \widehat{V}_{1 i}\left(\alpha, \beta_{0}\right)\right| \\
& \geq \frac{\left|\lambda_{1}\right| \bar{U}_{1}^{2}(\alpha)}{1+\left|\lambda_{1}\right| \max _{1 \leq i \leq 2 n_{0}}\left|\widehat{V}_{1 i}\left(\alpha, \beta_{0}\right)\right|}-U_{1}(\alpha)
\end{aligned}
$$

combining this with Lemma 3 and (15), we have $\lambda_{1}=$ $O_{p}\left(n_{0}^{-1 / 3}\right)$. Similarly, we can show $\lambda_{2}=O_{p}\left(n_{0}^{-1 / 3}\right)$.

Proof of Proposition 1. By Lemma 5, we have

$$
\lambda_{1} \widehat{V}_{1 i}\left(\alpha, \beta_{0}\right)=o_{p}(1), \quad \lambda_{2} \widehat{V}_{2 j}\left(\alpha, \beta_{0}\right)=o_{p}(1) .
$$

Since (9) is equal to

$$
U_{1}(\alpha)-\lambda_{1} \bar{U}_{1}^{2}(\alpha)+\frac{1}{2 n_{0}} \lambda_{1}^{2} \sum_{i=1}^{2 n_{0}} \frac{\widehat{V}_{1 i}^{3}\left(\alpha, \beta_{0}\right)}{1+\lambda_{1} \widehat{V}_{1 i}\left(\alpha, \beta_{0}\right)}=0
$$

we have

$$
\lambda_{1}=\left[\bar{U}_{1}^{2}(\alpha)\right]^{-1} U_{1}(\alpha)+o_{p}\left(n_{0}^{-1 / 3}\right) .
$$

Similarly,

$$
\lambda_{2}=\left[\bar{U}_{2}^{2}(\alpha)\right]^{-1} U_{2}(\alpha)+o_{p}\left(n_{0}^{-1 / 3}\right) .
$$

Denote $l_{1}\left(\alpha, \beta_{0}\right)=2 \sum_{i=1}^{2 n_{0}} \log \left\{1+\lambda_{1} \widehat{V}_{1 i}\left(\alpha, \beta_{0}\right)\right\}, l_{2}\left(\alpha, \beta_{0}\right)=$ $2 \sum_{j=1}^{n_{1}} \log \left\{1+\lambda_{2} \widehat{V}_{2 j}\left(\alpha, \beta_{0}\right)\right\}$, and set $\alpha=\alpha_{0}+n_{0}^{-1 / 3}$. Applying Taylor expansion to $l_{1}\left(\alpha, \beta_{0}\right)$,

$$
\begin{aligned}
l_{1}\left(\alpha, \beta_{0}\right) & =2 \sum_{i=1}^{2 n_{0}} \log \left\{1+\lambda_{1} \widehat{V}_{1 i}\left(\alpha, \beta_{0}\right)\right\} \\
& =2 \lambda_{1} \sum_{i=1}^{2 n_{0}} \widehat{V}_{1 i}\left(\alpha, \beta_{0}\right)-\lambda_{1}^{2} \sum_{i=1}^{2 n_{0}} \widehat{V}_{1 i}^{2}\left(\alpha, \beta_{0}\right)+o_{p}\left(n_{0}^{1 / 3}\right)
\end{aligned}
$$




$$
\begin{aligned}
= & 2 n_{0}\left\{\bar{U}_{1}^{2}(\alpha)\right\}^{-1} U_{1}^{2}(\alpha)+o_{p}\left(n_{0}^{1 / 3}\right) \\
= & 2 n_{0}\left\{\bar{U}_{1}^{2}\left(\alpha_{0}\right)+o_{p}(1)\right\}^{-1} \\
& \times\left\{U_{1}\left(\alpha_{0}\right)+\left(U_{1}\left(\alpha^{*}\right)+1\right)\left(\alpha-\alpha_{0}\right)\right\}^{2}+o_{p}\left(n_{0}^{1 / 3}\right) .
\end{aligned}
$$

By Lemma 3 and the fact that $U_{1}(\alpha) \rightarrow 0$ uniformly in $\left\{\alpha:\left|\alpha-\alpha_{0}\right| \leq n_{0}^{-1 / 3}\right\}$, we obtain

$$
l_{1}\left(\alpha, \beta_{0}\right) \geq C n_{0}^{1 / 3}
$$

with probability tending to one. On the other hand,

$$
l_{1}\left(\alpha_{0}, \beta_{0}\right)=2 n_{0}\left\{\bar{U}_{1}^{2}\left(\alpha_{0}\right)\right\}^{-1} U_{1}^{2}\left(\alpha_{0}\right)+o_{p}(1)=O_{p}(1) .
$$

Thus, as $n_{0} \rightarrow \infty$, with probability tending to one, it holds that $l_{1}\left(\alpha_{0}+n_{0}^{-1 / 3}, \beta_{0}\right)>l_{1}\left(\alpha_{0}, \beta_{0}\right)$. Similarly, we can show that $l_{1}\left(\alpha_{0}-n_{0}^{-1 / 3}, \beta_{0}\right)>l_{1}\left(\alpha_{0}, \beta_{0}\right)$. Also, we can prove $l_{2}\left(\alpha_{0} \pm n_{0}^{-1 / 3}, \beta_{0}\right)>l_{2}\left(\alpha_{0}, \beta_{0}\right)$ in the same manner.

Since $l_{1}\left(\alpha, \beta_{0}\right)$ and $l_{2}\left(\alpha, \beta_{0}\right)$ are continuous in $\left[\alpha_{0}-\right.$ $\left.n_{0}^{-1 / 3}, \alpha_{0}+n_{0}^{-1 / 3}\right], l\left(\alpha, \beta_{0}\right)$ attains its minimum in the interior of $\left[\alpha_{0}-n_{0}^{-1 / 3}, \alpha_{0}+n_{0}^{-1 / 3}\right]$, denoted by $\hat{\alpha}$.

Proof of Theorem 1. Put $\hat{\lambda}_{i}=\lambda_{i}(\hat{\alpha}), i=1,2$. Then

$$
Q_{j n_{0} n_{1}}\left(\hat{\alpha}, \hat{\lambda}_{1}, \hat{\lambda}_{2}\right)=0, \quad j=1,2,3 .
$$

By Taylor expansion, we have

$$
\begin{aligned}
0= & Q_{j n_{0} n_{1}}\left(\alpha_{0}, 0,0\right) \\
& +\frac{\partial}{\partial \lambda_{1}} Q_{j n_{0} n_{1}}\left(\alpha^{*}, \lambda_{1}^{*}, \lambda_{2}^{*}\right) \hat{\lambda}_{1}+\frac{\partial}{\partial \lambda_{2}} Q_{j n_{0} n_{1}}\left(\alpha^{*}, \lambda_{1}^{*}, \lambda_{2}^{*}\right) \hat{\lambda}_{2} \\
& +\frac{\partial}{\partial \alpha} Q_{j n_{0} n_{1}}\left(\alpha^{*}, \lambda_{1}^{*}, \lambda_{2}^{*}\right)\left(\hat{\alpha}-\alpha_{0}\right)
\end{aligned}
$$

where $\alpha^{*}$ lies between $\hat{\alpha}$ and $\alpha_{0}, \lambda_{l}^{*}$ lies between $\hat{\lambda}_{l}$ and $\lambda_{l}$. Using Lemma 5 , it can be checked that

$$
\begin{aligned}
& \lim _{n_{0}} \rightarrow \infty \frac{\partial}{\partial \lambda_{1}} Q_{1 n_{0} n_{1}}\left(\alpha, \lambda_{1}, \lambda_{2}\right) \\
&=\lim _{n_{0} \rightarrow \infty} \frac{1}{2 n_{0}} \sum_{i=1}^{2 n_{0}} \frac{-\widehat{V}_{1 i}^{2}\left(\alpha, \beta_{0}\right)}{\left(1+\lambda_{1} \widehat{V}_{1 i}\left(\alpha, \beta_{0}\right)\right)^{2}} \\
&=\lim _{n_{0} \rightarrow \infty} \frac{-1}{2 n_{0}} \sum_{i=1}^{2 n_{0}} \widehat{V}_{1 i}^{2}\left(\alpha, \beta_{0}\right) \\
&=-S_{1}^{2}, \\
& \lim _{n_{0}} \rightarrow \infty \frac{\partial}{\partial \lambda_{2}} Q_{1 n_{0} n_{1}}\left(\alpha, \lambda_{1}, \lambda_{2}\right)=0, \\
& \lim _{n_{0}} \rightarrow \infty \frac{\partial}{\partial \alpha} Q_{1 n_{0} n_{1}}\left(\alpha, \lambda_{1}, \lambda_{2}\right) \\
& \quad=\lim _{n_{0} \rightarrow \infty} \frac{1}{2 n_{0}} \sum_{i=1}^{2 n_{0}} \frac{\widehat{V}_{1 i}\left(\alpha, \beta_{0}\right)+1}{\left(1+\lambda_{1} \widehat{V}_{1 i}\left(\alpha, \beta_{0}\right)\right)^{2}} \\
&=1,
\end{aligned}
$$

$$
\begin{aligned}
& \lim _{n_{1} \rightarrow \infty} \frac{\partial}{\partial \lambda_{1}} Q_{2 n_{0} n_{1}}\left(\alpha, \lambda_{1}, \lambda_{2}\right)=0, \\
& \lim _{n_{1} \rightarrow \infty} \frac{\partial}{\partial \lambda_{2}} Q_{2 n_{0} n_{1}}\left(\alpha, \lambda_{1}, \lambda_{2}\right) \\
& =\lim _{n_{1} \rightarrow \infty} \frac{1}{n_{1}} \sum_{j=1}^{n_{1}} \frac{-\widehat{V}_{2 j}^{2}\left(\alpha, \beta_{0}\right)}{\left(1+\lambda_{2} \widehat{V}_{2 j}\left(\alpha, \beta_{0}\right)\right)^{2}} \\
& =-S_{2}^{2}, \\
& \quad \lim _{n_{1} \rightarrow \infty} \frac{\partial}{\partial \alpha} Q_{2 n_{0} n_{1}}\left(\alpha, \lambda_{1}, \lambda_{2}\right) \\
& \quad=-1, \\
& \lim _{1} \rightarrow \infty \\
& \lim _{n_{0} \rightarrow \infty} \frac{\partial}{n_{1}} \sum_{j=1}^{n_{1}} \frac{-\widehat{V}_{2 j}\left(\alpha, \beta_{0}\right)-1}{\left(1+\lambda_{2} \widehat{V}_{2 j}\left(\alpha, \beta_{0}\right)\right)^{2}} \\
& \lim _{3 n_{0} n_{1}}\left(\alpha, \lambda_{1}, \lambda_{2}\right)=1, \\
& n_{0} \rightarrow \infty \\
& \lim _{n_{0} \rightarrow \infty} \frac{\partial}{\partial \lambda_{2}} Q_{3 n_{0} n_{1}}\left(\alpha, \lambda_{1}, \lambda_{2}\right)=-\rho, \\
& \frac{\partial n_{0} n_{1}}{}\left(\alpha, \lambda_{1}, \lambda_{2}\right)=0 .
\end{aligned}
$$

All limits hold uniformly in $\left\{\alpha:\left|\alpha-\alpha_{0}\right| \leq n_{0}^{-1 / 3}\right\}$. Therefore, solving the equation (18), we have

$$
\begin{aligned}
\left(\begin{array}{c}
\hat{\lambda}_{1} \\
\hat{\lambda}_{2} \\
\hat{\alpha}-\alpha_{0}
\end{array}\right) & =-S_{n}^{-1}\left(\alpha^{*}, \lambda_{1}^{*}, \lambda_{2}^{*}\right)\left(\begin{array}{c}
Q_{1 n_{0} n_{1}}\left(\alpha_{0}, 0,0\right) \\
Q_{2 n_{0} n_{1}}\left(\alpha_{0}, 0,0\right) \\
0
\end{array}\right) \\
& =-S^{-1}\left(\begin{array}{c}
Q_{1 n_{0} n_{1}}\left(\alpha_{0}, 0,0\right) \\
Q_{2 n_{0} n_{1}}\left(\alpha_{0}, 0,0\right) \\
0
\end{array}\right)+o_{p}(1),
\end{aligned}
$$

where

$$
\begin{aligned}
& S_{n}\left(\alpha, \lambda_{1}, \lambda_{2}\right) \\
& \quad=\left(\begin{array}{lll}
\frac{\partial Q_{1 n_{0} n_{1}}\left(\alpha, \lambda_{1}, \lambda_{2}\right)}{\partial \lambda_{1}} & \frac{\partial Q_{1 n_{0} n_{1}}\left(\alpha, \lambda_{1}, \lambda_{2}\right)}{\partial \lambda_{2}} & \frac{\partial Q_{1 n_{0} n_{1}}\left(\alpha, \lambda_{1}, \lambda_{2}\right)}{\partial \alpha} \\
\frac{\partial Q_{2 n_{0} n_{1}\left(\alpha, \lambda_{1}, \lambda_{2}\right)}}{\partial \lambda_{1}} & \frac{\partial Q_{2 n_{0} n_{1}}\left(\alpha, \lambda_{1}, \lambda_{2}\right)}{\partial \lambda_{2}} & \frac{\partial Q_{2 n_{0} n_{1}}\left(\alpha, \lambda_{1}, \lambda_{2}\right)}{\partial \alpha} \\
\frac{\partial Q_{3 n_{0} n_{1}\left(\alpha, \lambda_{1}, \lambda_{2}\right)}}{\partial \lambda_{1}} & \frac{\partial Q_{3 n_{0} n_{1}\left(\alpha, \lambda_{1}, \lambda_{2}\right)}}{\partial \lambda_{2}} & \frac{\partial Q_{3 n_{0} n_{1}\left(\alpha, \lambda_{1}, \lambda_{2}\right)}}{\partial \alpha}
\end{array}\right) \\
& \stackrel{p}{\rightarrow} S:=\left(\begin{array}{ccc}
-S_{1}^{2} & 0 & 1 \\
0 & -S_{2}^{2} & -1 \\
1 & -\rho & 0
\end{array}\right)
\end{aligned}
$$

and thus

$$
S^{-1}:=\frac{1}{|S|}\left(\begin{array}{ccc}
-\rho & -\rho & S_{2}^{2} \\
-1 & -1 & -S_{1}^{2} \\
S_{2}^{2} & -\rho S_{1}^{2} & S_{1}^{2} S_{2}^{2}
\end{array}\right),
$$

where $|S|=S_{1}^{2} \rho+S_{2}^{2}$. So,

$$
\begin{aligned}
& \hat{\lambda}_{1}=\frac{\rho}{|S|}\left\{Q_{1 n_{0} n_{1}}\left(\alpha_{0}, 0,0\right)+Q_{2 n_{0} n_{1}}\left(\alpha_{0}, 0,0\right)\right\}+o_{p}(1), \\
& \hat{\lambda}_{2}=\frac{1}{|S|}\left\{Q_{1 n_{0} n_{1}}\left(\alpha_{0}, 0,0\right)+Q_{2 n_{0} n_{1}}\left(\alpha_{0}, 0,0\right)\right\}+o_{p}(1),
\end{aligned}
$$

300 B.-Y. Jing et al. 


$$
\begin{aligned}
\hat{\alpha}-\alpha_{0}= & \frac{-1}{|S|}\left\{S_{2}^{2} Q_{1 n_{0} n_{1}}\left(\alpha_{0}, 0,0\right)-\rho S_{1}^{2} Q_{2 n_{0} n_{1}}\left(\alpha_{0}, 0,0\right)\right\} \\
& +o_{p}(1) .
\end{aligned}
$$

The above gives that $\hat{\lambda}_{1}=\rho \hat{\lambda}_{2}+o_{p}(1)$. Furthermore,

$$
\sqrt{n_{1}}\left(\begin{array}{l}
Q_{1 n_{0} n_{1}}\left(\alpha_{0}, 0,0\right) \\
Q_{2 n_{0} n_{1}}\left(\alpha_{0}, 0,0\right)
\end{array}\right) \stackrel{d}{\rightarrow} N\left(\left(\begin{array}{l}
0 \\
0
\end{array}\right),\left(\begin{array}{cc}
\rho S_{1}^{2} & 0 \\
0 & S_{2}^{2}
\end{array}\right)\right),
$$

which implies that

$$
\begin{aligned}
l\left(\beta_{0}\right)= & 2\left\{\sum_{i=1}^{2 n_{0}} \log \left(1+\lambda_{1} \widehat{V}_{1 i}\left(\hat{\alpha}, \beta_{0}\right)\right)\right. \\
& \left.+\sum_{j=1}^{n_{1}} \log \left(1+\lambda_{2} \widehat{V}_{2 j}\left(\hat{\alpha}, \beta_{0}\right)\right)\right\} \\
= & 2 \sum_{i=1}^{2 n_{0}} \lambda_{1}(\hat{\alpha}) \widehat{V}_{1 i}\left(\hat{\alpha}, \beta_{0}\right)-\sum_{i=1}^{2 n_{0}} \lambda_{1}^{2}(\hat{\alpha}) \widehat{V}_{1 i}^{2}\left(\hat{\alpha}, \beta_{0}\right)+o_{p}(1) \\
& +2 \sum_{j=1}^{n_{1}} \lambda_{2}(\hat{\alpha}) \widehat{V}_{2 j}\left(\hat{\alpha}, \beta_{0}\right)-\sum_{j=1}^{n_{1}} \lambda_{2}^{2}(\hat{\alpha}) \widehat{V}_{2 j}^{2}\left(\hat{\alpha}, \beta_{0}\right)+o_{p}(1) \\
= & 2 n_{0} \hat{\lambda}_{1}^{2} \bar{U}_{1}^{2}(\hat{\alpha})+n_{1} \hat{\lambda}_{2}^{2} \bar{U}_{2}^{2}(\hat{\alpha})+o_{p}(1) \\
= & \left\{\left(\rho^{-1}+o(1)\right) \rho^{2} \bar{U}_{1}^{2}(\alpha)+\bar{U}_{2}^{2}(\alpha)\right\}\left(\sqrt{n_{1}} \hat{\lambda}_{2}\right)^{2}+o_{p}(1) \\
= & \left\{(\rho+o(1)) S_{1}^{2}+S_{2}^{2}\right\}\left(\sqrt{n_{1}} \hat{\lambda}_{2}\right)^{2}+o_{p}(1) \\
\stackrel{d}{\rightarrow} & \chi_{1}^{2} .
\end{aligned}
$$

\section{ACKNOWLEDGEMENTS}

The authors would like to thank the Editor, an Associate Editor and referees for their suggestions and criticisms which helped to improve this paper. Jing's research is partially supported by HK RGC grants HKUST6011/07P and HKUST6015/08P, HKUST6019/10P, the National Nature Science Foundation of China (71071155), the Fundamental Research Funds for the Central Universities, and the Research Funds of Renmin University of China (Grant No. 10XNL007), and in part by NSFC (Grant No. 71071155). Li's research is partially supported by the Fundamental Research Funds for the Central Universities (Grant No. lzujbky-2011-121). Zhou's research is partially supported by a grant R-155-000-116-112 at the National University of Singapore.

\section{Received 3 August 2011}

\section{REFERENCES}

Anderson, J. (1979). Robust inference using logistic models. Bull. Int. Statist. Inst. 2 35-53. MR0731556

Anderson, J. and Blair, V. (1982). Penalized maximum likelihood estimation in logistic regression and discrimination. Biometrika 69 123-136. MR0655677
Andrew, D. F. and Herzberg, A. M. (1985). Data: A Collection of Problems from Many Fields for the Student and Research Worker. Springer, New York.

Arvesen, J. (1969). Jackknifing U-statistics. Ann. Math. Statist. 40 2076-2100. MR0264805

Breslow, N. and Day, N. (1980). Statistical Methods in Cancer Research 1, The Analysis of Case-Control Studies. IARC, Lyon.

Breslow, N. (1996). Statistics in epidemiology: The case control study. J. Amer. Statist. Assoc. 91 14-28. MR1394064

Breslow, N., Robins J., and Wellner, J. (2000). On the semiparametric efficiency of logistic regression under case-control sampling. Bernoulli 6 447-455. MR1762555

Cameron, E. and Pauling, L. (1978). Supplemental ascorbate in the supportive treatment of cancer: Reevaluation of prolongation of survival times in terminal human cancer. Proc. Natl. Acad. Sci. U.S.A. 75, 4538-4542.

Chatterjee, N. and Carroll, R. (2005). Semiparametric maximum likelihood estimation exploiting gene-environment independent in case-control studies. Biometrika 92 399-418. MR2201367

Cox, T. and FERRY, G. (1991). Robust logistic discrimination. Biometrika 78 841-849. MR1147021

Fokianos, K. (2007). Density ratio model selection. Journal of Statistical Computation and Simulation 77 805-819. MR2409865

Gill, R., VArdi, Y., and Wellner, J. (1988). Large sample theory of empirical distributions in biased sampling models. Ann. Statist. 16 1069-1112. MR0959189

Godambe, V. (1960). An optimum property of regular maximum likelihood estimation. Ann. Math. Statist. 31 1208-1212. MR0123385

Hwang, S., Beaty, T., Panny, S., Street, N., Joseph, J., Gordon, S., McIntosh, I., and Francomano, C. (1995). Association study of transforming growth factor alpha (TGF $\alpha$ ) Taq1 polymorphism and oral clefts: Indication of gene-environment interaction in a population-based sample of infants with birth defects. Am. J. Epidemiology $141629-636$.

Jing, B., YuAn, J., and ZhOU, W. (2009). Jackknife empirical likelihood. J. Amer. Statist. Association 104 1224-1232. MR2562010

Modan, M., Hartge, P., et al. (2001). Parity, oral contraceptives and the risk of ovarian cancer among carriers and noncarriers of a BRCA1 or BRCA2 mutation. New Engl. J. Med. 345 235-240.

Owen, A. (1988). Empirical likelihood ratio confidence intervals for a single functional. Biometrika 75 237-249. MR0946049

Owen, A. (1990). Empirical likelihood ratio confidence regions. Ann. Statist. 18 90-120. MR1041387

Owen, A. (2001). Empirical Likelihood. Chapman \& Hall, London.

O'Neill, T. (1980). The general distribution of the error rate of a classification procedure with applications to the logistic regression discrimination. J. Am. Statist. Assoc. 75 154-160. MR0568586

Piegorsch, W., Weinberg, C. and Taylor, J. (1994). Nonhierarchical logistic models and case-only designs for assessing susceptibility in population based case-control studies. Statist. Med. 13 $153-162$.

Prentice, R. and Pyke, R. (1979). Logistic disease incidence models and case-control studies. Biometrika 66 403-411. MR0556730

QIN, J. (1993). Empirical likelihood in biased sample problems. Ann. Statist. 21 1182-1196. MR1241264

QIN, J. (1998). Inferences for case-control and semiparametric twosample density ratio models. Biometrika 85 619-630. MR1665814

QIN, J. and ZHANG, B. (1997). A goodness-of-fit test for logistic regression models based on case-control data. Biometrika 84 609-618. MR1603924

Schechtman, E. and Schechtman, G. (2002). On characterization of two-sample U-statistics. Statist. \& Probab. Lett. 58 53-59. MR1913308

SHI, X. (1984). The approximate independence of jackknife pseudovalues and the bootstrap methods. Journal of Wuhan Institute Hydra-Electric Engineering 2 83-90.

Umbach, D. and Weinberg, C. (1997). Designing and analyzing casecontrol studies to exploit independence of genotype and exposure. Statist. Med. 16, 1731-1743. 
VARDI, Y. (1982). Nonparametric estimation in the presence of length bias. Ann. Statist. 10, 616-620. MR0653536

WANG, Y. and CARroll, R. J. (1993). On robust estimation in logistic case-control studies. Biometrika 80 237-241. MR1225228

Weinberg, C. and Wacholder, S. (1993). Prospective analysis of case-control data under general multiplicative-intercept risk models. Biometrika 80 461-465. MR1243520

\section{Bing-Yi Jing}

Department of Mathematics

Hong Kong University of Science and Technology

Clear Water Bay, Kowloon

Hong Kong

E-mail address: majing@ust.hk

Zhouping $\mathrm{Li}$

School of Mathematics and Statistics

Lanzhou University

Lanzhou 730000

China

E-mail address: lizhp@lzu.edu.cn
Jing Qin

Biostatistics Research Branch

National Institute of Allergy and Infectious Diseases, NIH

Bethesda, MD 20892

USA

E-mail address: jingqin@niaid.nih.gov

Wang Zhou

Department of Statistics and Applied Probability

National University of Singapore

Singapore 117546

Singapore

E-mail address: stazw@nus.edu.sg 